\title{
The Relationship between Leadership and Communication Skills of Nurse Managers and the Organizational Citizenship Behaviors of Medical-Surgical Nurses and Nursing Assistants
}

\author{
Cheryl B, Cullen ${ }^{1} \&$ Pamela A. Gordon ${ }^{2, *}$ \\ ${ }^{1}$ College of Health Sciences, Walden University, Boonton, NJ, USA \\ ${ }^{2}$ Academic Affairs, University of Phoenix, Pembroke Pines, FL., USA \\ *Corresponding author: 1470 SW 164th Avenue Pembroke Pines, Florida 33027, USA. Tel: 1-954-614-4294. E-mail: \\ pam.gordon@phoenix.edu
}

Received: March 10, 2014

Accepted: March 26, $2014 \quad$ Online Published: April 18, 2014

doi:10.5430/mos.v1n2p23

URL: http://dx.doi.org/10.5430/mos.v1n2p23

\begin{abstract}
This quantitative research study, with a correlation design, used an online survey to determine if a positive correlation existed between the leadership and communication skills of nurse managers and the organizational citizenship behaviors of the nurses and nursing assistants who work on the medical-surgical nursing units of a community hospital in Northern New Jersey. The predictor variables were the employee's perceptions of their nurse manager leadership and communication skills and the self-reported organizational citizenship behaviors of the registered nurses and nursing assistants. Results demonstrated a moderately positive, statistically significant correlation, $r .35$ and $p$ value .000, between the leadership and communication skills of the nurse manager and the self-reported organizational citizenship behaviors of the registered nurses and nursing assistants. A leadership implication from this study is that nurse managers who have effective leadership and communication skills have a positive impact on the organizational citizenship behaviors of the nursing team.
\end{abstract}

Keywords: leadership; communication; organizational citizenship; nurse managers; quantitative research; correlation design

\section{Introduction}

\subsection{Problem}

Generous compensation packages and sign-on bonuses may attract nurses to an organization, but the ability to retain proficient, dedicated nurses is a challenge (Davis, Ward, Woodall, Shultz, \& Davis, 2007). Employer-employee relationships have a direct effect on employee commitment, work performance, and job engagement. The reasons often cited by nurses for leaving a nursing unit or health care organization include heavy workloads, harassment from colleagues, patients, or physicians, inflexible work schedules, the inability to provide quality care, and poor communication with the nurse manager (Kowalski et al, 2010; Spence Laschinger, Leiter, Day, \& Gilin, 2009).

\subsection{Importance of the Problem}

Recent changes in Centers for Medicare and Medicaid Services (CMS) standards tie quality indicators and patient satisfaction metrics directly to reimbursement, which reinforces the need for hospital leaders to ensure adequate staffing, reduction of agency staff usage, reduced staff turnover, and quality patient care (Averhill, Vertrees, McCullough, Hughes, \& Goldfield, 2006). A culture of transparency and caring that allows the nursing team to provide safe, quality, patient care, and retain seasoned nursing team members is a priority for hospital administrators and nurse managers (Cullen, 1999). The ability of the manager to communicate in a clear, consistent manner promotes an open and honest workplace culture.

\subsection{Relevant Scholarship}

A lack of synergy between a manager and employee is often cited by researchers as the number one reason an employee leaves (Branham, 2005). Empirical research, in venues other than medical-surgical nursing units, suggests that 
employees who are treated with respect, kept informed, and have positive two-way communication with the leadership team display improved productivity, increased job satisfaction, and staff retention (Boyle, Bott, Hansen, Woods, \& Taunton, 1999; Hogan, Moxham, \& Dywer, 2007). Two Australian studies suggested that further study is necessary to determine if a relationship exists between the characteristics of the nurse manager and the organizational loyalty of the acute care nurse as demonstrated by intent to leave the organization (Hogan, Moxham, \& Dywer, 2007). Therefore, a gap in the literature exists to determine if a correlation between nurse manger leadership and communication skills and employee job satisfaction and organizational citizenship behaviors in acute care, medical-surgical nurses and nursing assistants in the United States.

Early empirical research by Smith, Organ, and Near (1983) defines organizational citizenship behavior as employee social behaviors beyond the organizational policies and job descriptions that set the tone of the culture. The leader is the role model of organizational citizenship behavior through staff engagement with mentorship, coaching, and empathy. The social exchange between the leader and the subordinates defines the expected cultural norm (Smith et al., 1983). The ability of the team to produce effective work and handle an unexpected crisis is a measure of the level of employee empowerment and organizational citizenship behavior (Moideencutty, 2005).

Leader-member exchange is the relationship between the supervisor and the employee. A mentor relationship based on trust and open communication will result in positive leader-member exchange and foster a culture of high levels of organizational citizenship behavior. Brannon et al. (2007) and Moideencutty (2005) suggest a positive correlation between employee perception of leader behaviors and employee commitment to work. A culture where managers are able to engage employees and make them feel valued improves the desire of the employee to exceed and support the team to succeed. These employees help each other complete work, have a positive attitude toward the organization, and are highly productive.

Effective communication with employees on a regular basis, both formally and informally informs employees of organizational goals, policies, and personal progress so that the work of the organization is accomplished. If the employees do not have regular contact and communication with supervisors, confusion may result. Formal communication through organizational policies, mission, vision, and employee feedback provide the groundwork for the work the employee must accomplish. Informal communication is necessary to remove employee obstacles, which may impede work, ensure departments within the organization are working well together, and address employee innovations in a timely manner (Johlke \& Duhan, 2000; Wilson, 2006). Role modeling of open communication by the manager sets the cultural norm for employees who will behave in the same manner.

Organizational citizenship behaviors for the purpose of this research were measured by employee responses in the Supervisor Leadership Communication Inventory by response to questions regarding purposeful absenteeism and disregard of organizational policies, lack of team work, and intent to leave, Leadership behaviors measured in the SLCI tool included poor planning, micromanagement, and inconsistent behaviors. Communication behaviors measured included mentoring, consistent feedback, and openness to new ideas.

\subsection{Hypotheses}

This study examined the relationship between the leadership and communication skills of nurse managers and the organizational citizenship behaviors of acute care medical-surgical nurses and nursing assistants. The following hypotheses were the bases for this study. Hypothesis one suggests a significant relationship exists between nursing team organizational citizenship behavior and nurse manager leadership behavior. Hypothesis 2 suggests a significant relationship exists between nursing team organizational citizenship behavior and nursing manager communication behaviors. Hypotheses 3 suggests a significant relations exists between the combined independent variables, nurse manager leadership and communication behaviors and the organizational citizenship behavior or the nursing staff. Hypothesis 4 suggests a significant relationship between the independent and dependent variables and the effect of specific demographic variables.

\section{Method}

The purpose of this quantitative, cross sectional, correlational research was to determine if a significant relationship exists between employee perception of nurse manager leadership and communication skills, the independent variables, and the organizational citizenship behavior of the nursing team, the dependent variable. Specific, self-reported employee citizenship behaviors considered for this research were loyalty, compliance with organizational policies, productivity, intent to leave, empowerment, trust, and innovation. The Supervisor Leadership and Communication Inventory (SLCI) was used to explore the possible relationship (Rouse, 2009). The IRB at the University of Phoenix 
approved this research.

Statistical analysis was performed to calculate the frequency and percentage of gender and age of the registered nurses and nursing assistants who participated in the study. Additionally, the study sample was sorted by job category and hours worked, length in job, and shift worked. Descriptive statistics were calculated sorting the SCLI questions by construct and employee response. The four constructs of manager leadership skills, manager communication skills, combined manager leadership and communication skills, and employee organizational citizenship behaviors. Minimum score, maximum score, mean, and standard deviation of each construct was calculated.

Using Minitab ${ }^{\circledR}$ software, Pearson correlation $r$ and $p$ values were calculated to determine if a significant relationship exists for each construct: manager leadership skills, manager communication skills, combined manager leadership, and communication skills. Pearson correlation $r$ and $p$ values were calculated to determine if a significant relationship exists between nurse manager leadership and communication skills and study population demographic variables. The demographic variables included job category, registered nurse or nursing assistant, gender, age, length in job category, shift worked, and hours worked, full-time, part-time, or per diem. Registered nurses and nursing assistants employed on medical-surgical units of a nonprofit acute care community hospital system in Northern New Jersey participated in the research study. The age range of the participants varied and all participants were over 18 years old. The participants worked full-time, part-time, or per diem. No outside agency staff was permitted to participate. The nurse managers, permanent charge nurses, and directors of nursing did not participate in the research study.

\subsection{Sampling Frame}

A power analysis to prevent Type I and Type II errors to reject or accept the null hypothesis was necessary to determine sample size. The sample size for correlation analysis with .80 effect size at $\alpha .05$ required a sample size of 107 . The chief nurse officer $(\mathrm{CNO})$ sent an introductory e-mail to the members of the nursing team eligible to participate in the survey. The entire roster of 252 registered nurses and nursing assistants, who worked on the medical-surgical nursing units, during the time frame of the study, received an invitation to participate to ensure an adequate number of survey participants. Study participants received the informed consent electronically prior to commencement of the survey. To ensure anonymity, the survey and consent were electronically encrypted by Survey Methods ${ }^{\circledR}$, the web-based company for the research study.

\subsection{Data Collection}

The research study used a 55 question Web-based collection tool (Survey Methods ${ }^{\circledR}$ ), which provided a convenient method for potential survey respondents. The survey population was technology savvy given the robust electronic medical record and intranet system of the organization. All employees had e-mail accounts and Internet access, which provided access to the research tool via a hyper-link.

\subsection{Validity and Reliability}

Rouse (2009), the author of the SCLI tool, provided a validity statement in the SCLI Users Manual. The 18 subject matter experts who evaluated the questions related to organizational culture made a determination that the 55 Likert-scaled survey questions were valid. The SCLI Climate Profile demonstrated a Cronbach's alpha of 0.98 reliability with regard to the supervisor category (Rouse, 2009).

\section{Results}

\subsection{Demographic Data}

The study population consisted of 85 registered nurses and 41 nursing assistants for a total of 126 respondents. The majority of the participants were female in both job category groups. The age range of $83 \%$ of the population of the nurses was evenly distributed from 26 to 55 years old. The age range of the $85 \%$ of the nursing assistants was 18 to 45 years old. Table 1 shows the survey participant demographic variables. 
Table 1. Survey Participant Demographic Variables

\begin{tabular}{lllll}
\hline Category & \multicolumn{2}{c}{ Registered Nurse } & \multicolumn{2}{c}{ Nursing Assistant } \\
\cline { 2 - 5 } & Frequency & Percentage & Frequency & Percentage \\
\hline Gender & & & & \\
Female & 84 & $99 \%$ & 36 & $88 \%$ \\
Male & 1 & $1 \%$ & 5 & $12 \%$ \\
& & & & \\
Age & & $12 \%$ & 18 & $44 \%$ \\
18 to 25 & 10 & $28 \%$ & 10 & $24 \%$ \\
26 to 35 & 24 & $27 \%$ & 7 & $17 \%$ \\
36 to 45 & 23 & $28 \%$ & 4 & $5 \%$ \\
46 to 55 & 24 & & & \\
\hline
\end{tabular}

Note: Total sample size of 126 includes registered nurses $(\mathrm{N}=85)$ and nursing assistants $(\mathrm{N}=41)$.

Job category demographics included hours-worked, length in job, and shift worked. In both job categories, $87 \%$ registered nurse and $71 \%$ nursing assistant, work full time. The majority, $72 \%$ of the registered nurses and $83 \%$ of the nursing assistants in the study, have been in their jobs 10 years or less. The majority of study participants, $54 \%$ registered nurses and $46 \%$ of nursing assistants in the study population worked the day shift. Table 2 contains the frequency and percent of the job category variables, hours worked, length in job, and shift worked sorted by job category.

Table 2. Survey Participant Job Category Variables

\begin{tabular}{lcccc}
\hline Category & \multicolumn{2}{c}{ Registered Nurse } & \multicolumn{2}{c}{ Nursing Assistant } \\
& Frequency & Percentage & Frequency & Percentage \\
\hline Hours-worked & & & & \\
Full-time & 74 & $87 \%$ & 29 & $71 \%$ \\
Part-time & 8 & $9 \%$ & 3 & $7 \%$ \\
Per-diem & 3 & $4 \%$ & 9 & $22 \%$ \\
& & & \\
Length in Job & & & & \\
Less than 1 year & 10 & $12 \%$ & 4 & $10 \%$ \\
1 to 4 years & 30 & $35 \%$ & 21 & $51 \%$ \\
5 to 10 years & 21 & $25 \%$ & 9 & $5 \%$ \\
11 to 15 years & 5 & $5 \%$ & 2 & $2 \%$ \\
16 to 20 years & 7 & $8 \%$ & 1 & $10 \%$ \\
21 years or greater & 12 & $14 \%$ & 4 & \\
& & & & \\
Shift Worked & & & & \\
Days & 46 & $54 \%$ & 19 & $17 \%$ \\
Evenings & 4 & $5 \%$ & 7 & $37 \%$ \\
Nights & 35 & $31 \%$ & 15 & \\
\hline
\end{tabular}

Note: Total sample size of 126 includes registered nurses $(\mathrm{N}=85)$ and nursing assistants $(\mathrm{N}=41)$.

\subsection{Statistical Results}

The respondent survey responses were collected in the Survey Methods ${ }^{\circledR}$ software and exported to Microsoft Excel. Eight of the Supervisor Communication items, 12 of the Supervisor Leadership items, 10 of the Employee Behavior items, and one of the Organizational Outcomes items were reverse scored. The question responses were on a five-point Likert scale. The average minimum, maximum, mean and standard deviation calculations were performed for each construct. Table 3 illustrates the results. 
Table 3. Descriptive Statistics for Each Construct

\begin{tabular}{lccccc}
\hline Construct & $n$ & Minimum & Maximum & M & SD \\
\hline Manager Leadership Skills & 126 & 1.67 & 4.27 & 3.29 & 0.4664 \\
Manager Communication Skills & 126 & 2.00 & 4.83 & 3.46 & 0.4993 \\
Combined (above) & 126 & 1.82 & 4.30 & 3.39 & 0.4568 \\
Employee Organizational & 126 & 2.30 & 4.21 & 3.54 & 0.3544 \\
Citizenship Behavior & & & & & \\
\hline
\end{tabular}

\subsection{Hypothesis Testing}

The strongest correlation of the four hypotheses tested was hypothesis two (H2). H2 tested the relationship between manager communication skills and employee citizenship behaviors. The data result was a positive correlation, $r .38$, and $\mathrm{p}$ value .000 , statistically significant. The null hypothesis was rejected. Hypothesis three $(\mathrm{H} 3)$ tested the relationship between the combined nurse manager leadership and communication skills and employee citizenship behaviors. The data result was a positive correlation $r .35, \mathrm{p}$ value .000 . The data suggested that as employee perception of positive manager leadership and communication skills increases, employee citizenship behaviors also increase. Hypothesis one (H1) tested the relationship between manager leadership skills and employee citizenship behaviors. Manager leadership and employee citizenship had a positive correlation, $r=.26, \mathrm{p} .003$. The null hypothesis was rejected.

The data results suggested that increased employee perception of nurse manager communication skills has a greater influence than employee perception of leadership skills on employee citizenship behavior. The results support the work of Studer (2003) regarding the importance of clear manager communication to ensure employees feel valued and have a feeling of belonging.

Hypothesis four tested the effect on the correlation between employee perception of nurse manager leadership and communication skills and employee organizational citizenship behaviors. The following demographic groups had a positive correlation $r$ and statistically significant $p$ value: registered nurse, nursing assistant, female gender, age group 18 to 25 years, age group 46 to 55 years, day shift, night shift, full time and part time, and all length in job categories except $<1$ year. Demographic populations that were not statistically significant due to a small population size were male gender, age group 56 years and older, and evening shift employees. The null hypothesis was not rejected because not all demographic categories were not statistically significant, even though there was a positive correlation $r$. Pearson correlation $r$ results and $p$ values for each demographic category follows:

\begin{tabular}{lccc}
\hline Job Category & Pearson correlation $\mathrm{r}$ & $\mathrm{p}$ value & Significant relationship? \\
\hline Registered nurse & .3386 & .000 & yes \\
Nursing assistant & .3762 & .001 & yes \\
Gender female & .35 & .000 & yes \\
Gender male & .67 & .146 & no \\
Age 18-25 years & .54 & .003 & yes \\
Age 26-35 years & .16 & .100 & no \\
Age 36-45 years & .26 & .158 & no \\
Age 46 to 55 years & .52 & .004 & yes \\
Age 55 years and older & .21 & .100 & no \\
Length in job $<1$ year & .67 & .146 & no \\
Length 1 to 4 years & .95 & .000 & yes \\
Length 5 to 10 years & .94 & .000 & yes \\
Length 11 to 15 years & .98 & .000 & yes \\
Length 16 to 20 years & .99 & .000 & yes \\
Length 21 years and & .96 & .000 & yes \\
Day shift & .40 & .001 & yes \\
Evening shift & .40 & .168 & no \\
Night shift & .30 & .038 & yes \\
Full time & .37 & .000 & yes \\
Part time & .19 & .000 & yes \\
Per diem & 14 & .662 & no \\
\hline
\end{tabular}




\section{Discussion}

The research results suggested nurse manager communication has a greater influence on employee citizenship behavior than nurse manager leadership skills within the study population of medical-surgical registered nurses and nursing assistants. $\mathrm{H} 2$, which tested the construct of the effect of nurse manager communication skills on employee citizenship behaviors, had a moderately strong correlation. Although this result does not provide evidence of causality between the predictor variable nurse manager communication and employee citizenship behavior, the result is statistically significant and is supported in the non-medical literature (Novicevic et al., 2005). Medical-surgical nurses and nursing assistants rely on effective communication between each other to ensure safe patient care. Open, transparent communication between the nurse manager and the nursing team may improve staff reporting of patient concerns, near-miss events, or staff frustrations and concerns (Grindel, 2004).

The combined construct of nurse manager leadership and communication skills positively correlated to employee organizational citizenship behavior compared to nurse manager leadership skills, which was weakly positive. This research supports the evidence from the non-medical literature regarding a positive correlation between the effect of manager behavior and leadership skills on employee citizenship behaviors (Smith, 1975). Nurse managers must be able to communicate, provide coaching, and act as advocates for the staff to ensure adequate resources to care for patients. The research informs medical-surgical nurse managers that their communication and leadership skills may influence the ability of the team to mature over time. This study adds to the body of literature with regard to the behaviors of medical-surgical registered nurses and nursing assistants.

Further research to compare results among teaching hospitals, unionized hospitals, and magnet designated hospitals may provide information to contribute to the body of nursing leadership knowledge and to determine if causality exists. A qualitative study that provides the respondents the opportunity to write comments and give examples of positive and negative interactions may provide opportunities for leaders to understand why. Generational considerations and themes may also provide nurse leaders information regarding the ability of nursing mangers to effectively communicate with the nursing team. Emerging themes could provide information for specific training programs and coaching for nurse managers. Expanding the survey to nurse leaders may provide an interesting comparison to determine if a positive correlation exists between the nurse manager and nursing staff responses.

Communication and leadership skills have an effect on employee citizenship behaviors, which includes staff satisfaction and retention. Decreasing registered nurse and nursing assistant turnover is a key initiative for healthcare leaders. Ensuring nurse managers have the communication skills necessary to effectively communicate with all members of the medical-surgical nursing team is essential and promotes a safe patient care environment. The study suggests evidence that nurse leader communication skills have an impact on employee citizenship behaviors. Further study is necessary to infer causality.

\section{References}

Averhill, R., Vertrees, J., McCullough, E., Hughes, J., \& Goldfield, N. (2006). Redesigning Medicare inpatient PPS to adjust payment for post-admission complications. Health Care Financing Review, 27(3), 83-93.

Boyle, D., Bott, M., Hansen, H., Woods, T., \& Taunton, R. (1999). Manager's leadership and critical care nurses' intent to stay. American Journal of Critical Care, 8(6), 361-371.

Branham, L. (2005). The 7 hidden reasons employees leave. New York: American Management Association.

Cullen, K. (1999). Strong leaders strengthen retention. Nursing Management, 30(5), 27-28. Retrieved from http://abi/informglobal

Davis, B., Ward, C., Woodall, M., Shultz, S., \& Davis, H. (2007). Comparison of job satisfaction between experienced medical-surgical nurses and experienced critical care nurses. MEDSURG Nursing, 16(5), 311-316.

Grindel, C. (2004). The leadership role of medical-surgical nurses: What is it? MEDSURG Nursing, 12(5), 361-362.

Hogan, P., Moxham, L., \& Dywer, T. (2007). Human resource management strategies for the retention of nurses in acute care settings in hospitals in Australia. Contemporary Nurse: A Journal of the Australian Nursing Profession, 24(12), 189-199. http://dx.doi.org/10.5172/conu.2007.24.2.189

Johlke, M., \& Duhan, D. (2000). Supervisor communication practices and service employee job outcomes. Journal of Service Research, 3(2), 154-165. http://dx.doi.org/10.1177/109467050032004

Kowalski, C., Ommen, O., Driller, E., Ernstmann, N., Wirtz, M., Köhler, T., \& Pfaff, H. (2010, June). Burnout in 
nurses: The relationship between social capital in hospitals and emotional exhaustion. Journal Clinical Nursing, 19(11/12), 1654-1663. http://dx.doi.org/10.1111/j.1365-2702.2009.02989.x

Moideenkutty, U. (2005). Organizational citizenship behavior and social exchange: A study of the effects of sources of positive benefits. Journal of Organizational Culture, Communications, and Conflict, 9(1), 1-10.

Novicevic, M., Davis, W., Dorn, F., Buckley, M., \& Brown, J. (2005). Barnard on conflicts of responsibility: Implications for today's perspectives on transformational and authentic leadership. Management Decision, 43(10), 1396-1409. http://dx.doi.org/10.1108/00251740510634930

Rouse, R. (2009). Supervisor leadership and communication inventory user's manual. Retrieved from http://www.drrichschuttler.com/slci-mini-test.htm

Smith, B., Organ, D., \& Near, J. (1983). Organizational citizenship behavior: Its nature and antecedents. Journal of Applied Psychology, 68(4), 653-663. http://dx.doi.org/10.1037/0021-9010.68.4.653

Smith, E. B. (1975). Chester Barnard's concept of leadership. Educational Administration Quarterly, 11(3), 37-48.

Spence Laschinger, H., Leiter, M., Day, A., \& Gilin, D. (2009, April). Workplace empowerment, incivility, and burnout: Impact in staff nurse recruitment and retention outcomes. Journal of Nursing Management, 17(3), 302-311. http://dx.doi.org/10.1111/j.1365-2834.2009.00999

Studer, Q. (2003). Hardwiring excellence: Purpose, worthwhile work, making a difference. Gulf Breeze, FL: Fire Starter Publishing. 